technique seems, however, to carry a higher risk of perioperative bleeding.

Our new inclusion technique combines several advantages. All the sutures are placed above the aortic anulus, facilitating the surgical approach, as with the Yacoub procedure. The aortic root is preserved, and therefore the spatial organization of the three leaflets and their commissures is minimally disturbed, facilitating understanding and reconstruction. The size of the Dacron tube is selected solely on the basis of the measured diameter of the aortic anulus and not on mathematical formulas, taking into account the height of the leaflets and the length of their free margins. Hemostasis is ensured by the large bites of the running suture and furthermore by enclosing all the sutures within the proximal aortic root. Finally, although the number of patients is still small, there have been no instances of postoperative aortic valve regurgitation.

Among the three operations described for preserving the aortic valve, only the David procedure could remodel the aortic anulus. The Yacoub procedure and the present operation are not designed to reduce the aortic anulus and therefore share the same limitations concerning annuloaortic ectasia and Marfan syndrome.

In conclusion, the Dacron tube inclusion technique seems promising. Compared with the other techniques, it associates the greater facility of a supra-annular suture line, an easier reconstruction of the native aortic valve, and a secure hemostasis. More cases will be needed to confirm the validity of this novel approach.
Table I. Patient demographics and operative data

\begin{tabular}{lc}
\hline No. of patients & 5 \\
Age (y) & $46-67$ \\
Preop aortic insufficiency & $1+$ to $3+$ \\
Marfan syndrome & 0 \\
Bicuspid valve & 1 \\
Associated procedures & \\
$\quad$ Coronary grafting & 2 \\
$\quad$ Arch replacement & 1 \\
$\quad$ Mitral repair & 1 \\
Aortic crossclamp (min) & $95 \pm 41$ \\
CPB time (min) & $115 \pm 25$ \\
Dacron diameter (mm) & $26-32$ \\
ICU bleeding (mL) & $730 \pm 520$ \\
Postop aortic regurgitation & 0 to trace \\
Deaths & 0 \\
Follow-up & 2 mo to 3 y \\
Reoperation & 0
\end{tabular}

$C P B$, Cardiopulmonary bypass; $I C U$, intensive care unit.

\section{REFERENCES}

1. David TE, Christopher MF. An aortic valve-sparing operation for patients with aortic incompetence and aneurysms of the ascending aorta. J Thorac Cardiovasc Surg 1992;103:617-22.

2. Yacoub MH, Gehle P, Chandrasekaran V, Birks EJ, Child A, Radley-Smith R. Late results of a valve-preserving operation in patients with aneurysms of the ascending aorta and root. J Thorac Cardiovasc Surg 1998:115:1080-90.

3. David TE. Aortic root aneurysms: Remodeling or composite replacement? Ann Thorac Surg 1997;64:1564-8.

\title{
REVERSAL OF SPINAL CORD ISCHEMIA RESULTING FROM AORTIC DISSECTION
}

\author{
Duncan A. Killen, MD, Charles L. Weinstein, MD, and William A. Reed, MD, Kansas City, Mo
}

Spinal cord ischemic injury is one of the hazards of acute aortic dissection. The following case report provides tentative evidence that, in certain instances, spinal cord ischemia resulting from acute aortic dissection may be completely reversed by therapeutic measures previously applied successfully to prevent and/or treat spinal cord ischemia incident to resection of thoracic and thoracoabdominal aortic aneurysms.

Clinical summary. A 57-year-old man had a history of longstanding medically controlled hypertensive cardiovascu-

From the Mid America Heart Institute, Saint Luke's Hospital, and the Departments of Surgery and Medicine (Section of Neurology), University of Missouri-Kansas City, School of Medicine, Kansas City, Mo.

Received for publication Jan 4, 2000; accepted for publication Jan 11, 2000.

Address for reprints: Duncan A. Killen, MD, 4320 Wornall, Suite 50II, Kansas City, MO 64111. lar disease. He had a brain stem stroke (dysconjugate gaze, ataxia, and dysarthria) $8 \frac{1}{2}$ years previously with complete recovery. At approximately 8 PM on February 6, 1999, he had the sudden onset of severe upper back pain. After being evaluated in the emergency department of his community hospital, he was transferred to Saint Luke's Hospital.

He was hypertensive $(183 / 68 \mathrm{~mm} \mathrm{Hg})$, all extremity pulses were normal by palpation, and the neurologic examination showed no abnormalities. Chest and abdominal com-

J Thorac Cardiovasc Surg 2000;119:1049-52.

Copyright (C) 2000 by The American Association for Thoracic Surgery

$0022-5223 / 2000 \$ 12.00+0 \quad \mathbf{1 2 / 5 4 / 1 0 5 7 4 8}$

doi:10.1067/mtc.2000.105748 


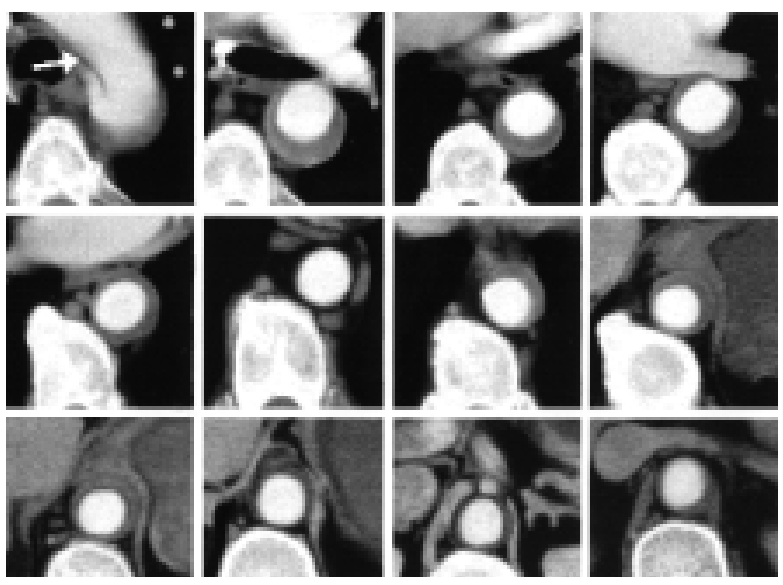

Fig 1. Serial 2-cm "cuts" of the computed tomographic scan of the descending thoracic and upper abdominal aorta revealing a contrast-filled aortic dissection limited to the region of the aortic isthmus (see arrow on cut No. 1) with an intramural (thrombosed) aortic dissection extending caudally to the level of the superior mesenteric artery.

puted tomographic scans (with intravascular contrast medium) revealed a limited secondary lumen of aortic dissection filled by contrast medium at the level of the aortic isthmus. Distally, the aortic wall was thickened by an intramural hematoma to the level of the origin of the superior mesenteric artery (Fig 1).

After transfer to the intensive care unit the patient was given intravenous drips of nitroprusside and labetalol. After 8 hours the labetalol drip was replaced with an esmolol drip. After the intravenous antihypertensive regimen was begun, the blood pressure was promptly brought under control and his pain gradually resolved completely. At approximately 8 AM ( 8 hours after admission to the intensive care unit), weakness of the left thigh and calf was noted. After progressing to also involve the right leg, the weakness stabilized. A neurologist detected total leg paraparesis with left leg muscle strength being graded 3/5 and right leg muscle strength 4/5 (5 being normal strength). Ankle reflexes were absent bilaterally. No sensory loss was detected over the lower torso or legs; there was no upper extremity weakness nor change in mentation. The weakness persisted unchanged.

After placement of a small catheter in the lumbar subarachnoid space, cerebrospinal fluid (CSF) drainage and an intravenous naloxone drip $\left(1 \mu \mathrm{g} \cdot \mathrm{kg}^{-1} \cdot \mathrm{h}^{-1}\right)$ were initiated approximately 8 hours after the onset of paraparesis. Two hours after initiation of CSF drainage the leg weakness began to resolve, and within the subsequent 6 hours the patient's neurologic status had returned to normal. He could stand without difficulty. The time course of these events is plotted in Fig 2. The following day, transesophageal echocardiography confirmed the presence of a blood-filled aortic dissection limited to a few centimeters in the region of the aortic isthmus with a thrombosed false lumen distally in the descending thoracic aorta.
The naloxone drip was discontinued after $2^{1 / 2}$ days, and CSF drainage was discontinued after approximately $3 \frac{1}{2}$ days. The patient's leg strength remained normal. His subsequent hospital course was unremarkable, and after being placed on an oral antihypertensive regimen he was discharged 3 days later receiving labetalol, nifedipine, and fosinopril. He has remained in neurologically intact condition during the subsequent 10 months.

Comment. The relationship of the spinal cord perfusion pressure to spinal cord ischemic injury has been elucidated during the past 4 decades by experimental studies using various animal preparations. ${ }^{1-4}$ Although other mechanisms of ischemic spinal cord damage undoubtedly exist, the importance of the residual spinal cord perfusion pressure during interruption of aortic and/or parietal segmental (intercostal and lumbar) arterial blood flow has been well documented by these studies.

The rarity of spinal cord ischemic injury during clinical "shock states" suggests that the spinal cord tolerates a rather low perfusion pressure for significant periods of time. Measurement of residual mean arterial pressure as lower thoracic intercostal arteries are ligated in animal preparations reveals that there is usually no spinal cord ischemic injury if the mean arterial back pressure remains above $35 \mathrm{~mm} \mathrm{Hg.}{ }^{3}$ Spinal cord ischemic damage usually occurs when the perfusion pressure remains below this level. ${ }^{3,5}$ When blood flow from an aortic segment to the spinal cord is interrupted, collateral circulation to the spinal cord can theoretically be increased by raising both the proximal and distal mean aortic pressures. This would enhance the blood supply to the region of the spinal cord excluded from direct arterial perfusion. ${ }^{5}$ Also, the perfusion pressure of a poorly perfused segment of spinal cord can be favorably affected by decreasing the pressure about the spinal cord by gravity drainage of the CSF, thus creating a low ambient pressure in the subarachnoid space that surrounds the spinal cord..$^{5}$ In the early 1960 s, drainage of the CSF was shown to minimize ischemic spinal cord injury in animals subjected to a standardized occlusion of the thoracic aorta. ${ }^{1,2}$ Also, the use of a topically applied vasodilator (papaverine) has been shown experimentally to minimize or prevent spinal cord ischemia by increasing collateral blood flow. ${ }^{6}$

The efficacy of hemodynamic manipulations during periods of compromised spinal cord perfusion has been more difficult to document clinically. ${ }^{7}$ The incidence of ischemic spinal cord damage during resection of thoracoabdominal aortic aneurysms can be correlated with certain nonhemodynamic parameters such as extensiveness of the aneurysm, the presence of associated aortic dissection, and the duration of aortic crossclamping, thereby confounding evaluation of the importance of the systemic or regional hemodynamic parameters. ${ }^{7-9}$ However, several large clinical experiences strongly suggest that a number of measures that may favorably modify the regional spinal cord perfusion are effective in minimizing or preventing spinal cord ischemic injury during resection of thoracoabdominal aortic aneurysms. ${ }^{4,6,8-11}$ These include intraoperative (extracorporeal) bypass, high cardiac 


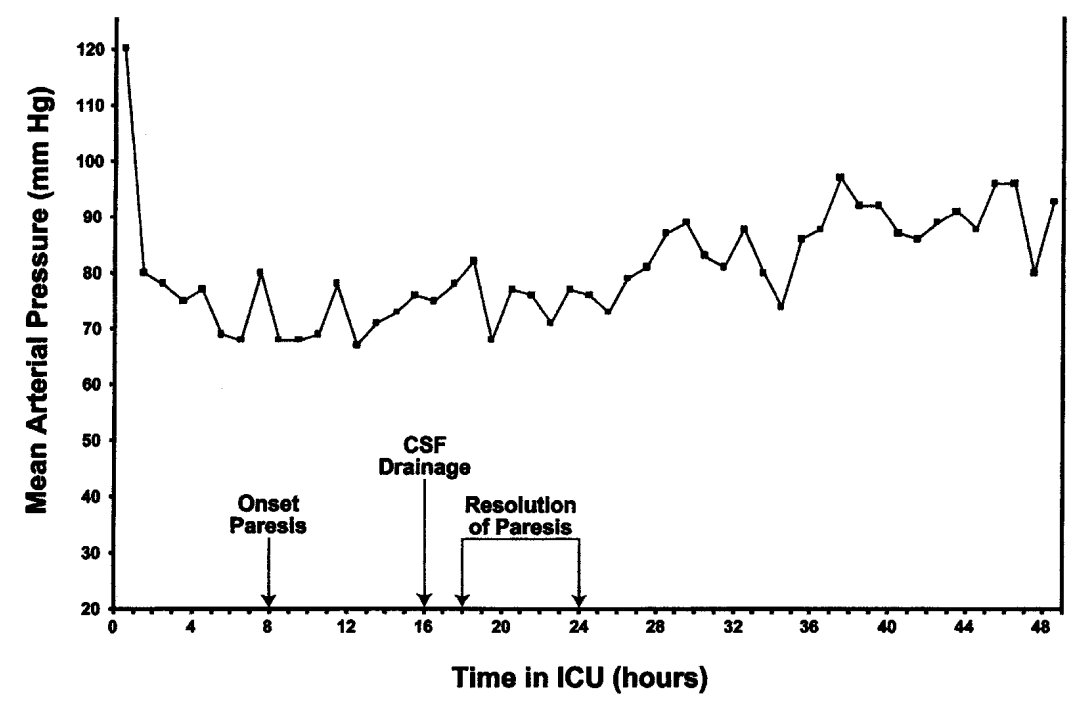

Fig 2. Course of mean arterial blood pressure, therapeutic interventions, and neurologic status after admission to the intensive care unit $(I C U)$.

output, high systemic blood pressure, sequential segmental aortic exclusion with early revascularization of segmental arteries, and CSF drainage, Also, the effectiveness of hypothermia and/or blockade of ischemia-induced neuroexcitatory impulses in protecting neurons, in particular those of the spinal cord, from ischemic damage has been demonstrated. ${ }^{12}$ Naloxone inhibits the effects of stress- and/or hypoxiainduced release of spinal cord excitatory endorphins that accentuate neuronal ischemic damage. ${ }^{8,9}$ Although this is thought to be the mechanism of naloxone protection against ischemic injury of the spinal cord, some other effect may be operative.

The incidence of delayed paraparesis and/or paraplegia after resection of thoracic or thoracoabdominal aneurysms is significant. ${ }^{4,7}$ The patient is neurologically normal on awakening from anesthesia, but lower body motor deficits develop during the first few postoperative days. In a number of such patients the initiation or reinstitution of CSF drainage and continuous intravenous infusion of naloxone has been followed by prompt reversal of the neurologic deficit. ${ }^{4,8,9} \mathrm{We}$ have observed this phenomenon on two occasions by reinitiating CSF drainage and intravenous naloxone infusion after late-onset spinal cord ischemic injury after resection of a thoracoabdominal aortic aneurysm.

Given the common etiology (regional hypoperfusion) of spinal cord ischemic injury that results from acute aortic dissection and injury occurring incident to resection of thoracoabdominal aortic aneurysms, it seems logical to apply the same promising therapies where feasible. Obviously, raising the systemic blood pressure in the presence of acute aortic dissection may be hazardous due to the immediate risk of further aortic dissection or aortic rupture. Ostensibly, CSF drainage and intravenous naloxone can be administered with low risk in the presence of acute aortic dissection. ${ }^{4}$ The opti- mal duration of these interventions is not known. Animal studies suggest that the collateral flow to the hypoperfused region improves over several hours and perhaps over a number of days. ${ }^{3,13}$ A 3-day course of CSF drainage, as recommended by Safi and associates, ${ }^{4}$ seems reasonable.

The incidence of spinal cord ischemia in patients with aortic dissection is approximately $4 \% .{ }^{14-16}$ Spinal cord ischemia is possibly more frequently associated with type III aortic dissections. ${ }^{15}$ The anterior spinal cord is often selectively or more severely involved, such that sensation is preserved in the lower extremities. The severity of the loss of motor function varies from mild to complete. The deficit may be permanent, may gradually improve, or in some instances may be transient, a syndrome perhaps best characterized as a spinal cord TIA (transient ischemic attack). ${ }^{14,15}$

Our patient's spinal cord ischemia might have resolved spontaneously, but we are struck by the rapidity of complete reversal of his leg weakness. The only way to prove or disprove the effectiveness of CSF drainage and systemic naloxone infusion in spinal cord ischemia resulting from acute aortic dissection is to analyze a large series of randomized cases. However, the low frequency of such cases in most physicians' experience makes such a study impractical. Because paraplegia is such a devastating complication and no treatment has been proved effective, we believe that CSF drainage and naloxone infusion should be given further clinical trial in the setting of spinal cord ischemia caused by acute aortic dissection.

\section{REFERENCES}

1. Miyamoto K, Ueno A, Wada T, Kimoto S. A new and simple method of preventing spinal cord damage following temporary occlusion of the thoracic aorta by draining cerebrospinal fluid. J Cardiovasc Surg 1960;1:188-97. 
2. Blaisdell FW, Cooley DA. The mechanism of paraplegia after temporary thoracic aortic occlusion and its relationship to spinal fluid pressure. Surgery 1962;51:351-5.

3. Killen DA, Adkins RB Jr. Studies of spinal arterial bed pressure following extensive mobilization of the aorta from the posterior parietes. J Thorac Cardiovasc Surg 1965;49:231-40.

4. Safi HJ, Miller CC 3d, Azizzadeh A, Iliopoulos DC. Observations on delayed neurologic deficit after thoracoabdominal aortic aneurysm repair. J Vasc Surg 1997;26:616-22.

5. McCullough JL, Hollier LH, Nugent M. Paraplegia after thoracic aortic occlusion: influence of cerebrospinal fluid drainage. J Vasc Surg 1988;7:153-60.

6. Svensson LG, Von Ritter CM, Groeneveld HT, Rickards ES, Hunter SJS, Robinson MF, et al. Cross-clamping of the thoracic aorta. Ann Surg 1986;204:38-47.

7. Crawford ES, Svensson LG, Hess KR, Shenaq SS, Costelli JS, Haziam JS, et al. A prospective randomized study of cerebrospinal fluid drainage to prevent paraplegia after high-risk surgery on the thoracoabdominal aorta. J Vasc Surg 1991;13:36-46.

8. Acher CW, Wynn MM, Hoch JR, Kranner PW. Cardiac function is a risk factor for paralysis in thoracoabdominal aortic replacement. J Vasc Surg 1998;27:821-30.

9. Acher CW, Wynn MM, Hoch JR, Popic P, Archibald J, Turnipseed WD. Combined use of cerebrospinal fluid drainage and naloxone reduces the risk of paraplegia in thoracoabdominal aneurysm repair. J Vasc Surg 1994;19:236-48.
10. de Haan P, Kalkman CJ, de Mol BA, Ubags LH, Veldman DJ, Jacobs MJH. Efficacy of transcranial motor-evoked myogenic potentials to detect spinal cord ischemia during operations for thoracoabdominal aneurysms. J Thorac Cardiovasc Surg 1997; 113:87-101.

11. Laschinger JC, Owen J, Rosenbloom M, Cox JL, Kouchoukos NT. Direct noninvasive monitoring of spinal cord motor function during thoracic aortic occlusion: use of motor evoked potentials. J Vasc Surg 1988;7:161-71.

12. Rokkas CK, Helfrich LR Jr, Lobner DC, Choi DW, Kouchoukos NT. Dextrorphan inhibits the release of excitatory amino acids during spinal cord ischemia. Ann Thorac Surg 1994;58:312-20.

13. Edwards RH, Killen DA. Prevention of spinal cord ischemia incident to extensive mobilization of the thoracic aorta from the posterior parietes. Am Coll Surg Forum 1964;15:285-7.

14. Zull DN, Cydulka R. Acute paraplegia: a presenting manifestation of aortic dissection. Am J Med 1988;84:765-70.

15. Spittell PC, Spittell JA Jr, Joyce JW, Tajik AJ, Edwards WD, Schaff HV, et al. Clinical features and differential diagnosis of aortic dissection: experience with 236 cases (1980 through 1990). Mayo Clin Proc 1993;68:642-51.

16. Tanaka T, Uemura K, Sugiura M, Ohishi H, Tomita M, Nagasaki $\mathrm{F}$, et al. Transient paraplegia caused by acute aortic dissection. Neurol Med Chir 1990;(Tokyo)30:54-8. 